University of Nebraska - Lincoln

DigitalCommons@University of Nebraska - Lincoln

Faculty Publications in Computer \& Electronics Electrical \& Computer Engineering, Department Engineering (to 2015)

9-2009

\title{
Effect of Sample Timing on LS Channel Estimation
}

Jason Uher

University of Nebraska-Lincoln, jasonuher@huskers.unl.edu

Tadeusz Wysocki

University of Nebraska-Lincoln, wysocki@uow.edu.au

Beata Wysocki

University of Nebraska-Lincoln, bwysocki2@unl.edu

Follow this and additional works at: https://digitalcommons.unl.edu/computerelectronicfacpub

Part of the Computer Engineering Commons

Uher, Jason; Wysocki, Tadeusz; and Wysocki, Beata, "Effect of Sample Timing on LS Channel Estimation" (2009). Faculty Publications in Computer \& Electronics Engineering (to 2015). 62.

https://digitalcommons.unl.edu/computerelectronicfacpub/62

This Article is brought to you for free and open access by the Electrical \& Computer Engineering, Department of at DigitalCommons@University of Nebraska - Lincoln. It has been accepted for inclusion in Faculty Publications in Computer \& Electronics Engineering (to 2015) by an authorized administrator of DigitalCommons@University of Nebraska - Lincoln. 


\title{
Effect of Sample Timing on LS Channel Estimation
}

\author{
Jason Uher ${ }^{1}$, Tadeusz Wysocki, Beata Wysocki \\ University of Nebraska-Lincoln \\ jjasonuher@huskers.unl.edu
}

\begin{abstract}
As block coding and intelligent receiver combining in multi-antenna systems moves from the theorectical into the physical domain, it is necessary to apply robust analysis to the problems encountered with the development of real radio systems that are often overlooked during theoretical development of methods and processes. One such problem is the lack of synchronization between the transmitter and receiver sampling clocks. This text attempts to analyze the significance of poorly correcting for differences in the transmit and receive sample clocks by simulating the BER of an Alamouti space-time coded system under a variety of sample rates and clock offsets. The results can be used to estimate the impact on link quality by a given sampler's estimation error using the empirical model given.
\end{abstract}

\section{INTRODUCTION}

The introduction of intelligent antenna combining and block coded MIMO systems have provided for a vast potential increase in the performance of wireless links which undergo complex multiplicative fading. Much theoretical work has been done to show that in the presence of a worst case fading, that has been drawn from a theoretical Rayleigh distribution, multi-antenna systems can provide a minimum of $3 \mathrm{~dB}$ in array gain for each additional antenna used [1], and can provide a significant improvement at higher SNRs when coding is used[2].

In most cases, practical radio issues such as carrier offset mismatches and a mistimed sample clock are ignored, even though such implementation issues can greatly affect the quality of the overall link. Some characterization of the error introduced by such practical limitations is useful for link budget design and system performance estimation.

In this paper we attempt to characterize the errors introduced by sampling offsets in an Alamouti space-time encoded system using least squares channel estimation. First, the system is simulated using a variety of symbol widths and offsets to provide a set of data that provides a relationship between the BER of the system and the offset of the estimated sample clock. Next, an empirical model of the extra error rate that has been introduced is extrapolated from the simulated data to provide an estimate of the error for a given combination of symbol widths and sample offsets. The rest of the paper is organized as follows; Section II details the theoretical model of the space-time system used to simulate the error rates for a given offset. Section III provides a detailed description of the problems introduced by symbol timing errors. Section IV presents the results from the simulation and provides an empirical model for the estimation of the BER for a given SNR, sample width, and sample offset.
Finally, Section V presents the empirical model derived from the simulation results.

\section{THEORETICAL AND SIMULATION SYSTEM MODEL}

In order to simulate a space-time block coded system under Rayleigh fading, the following mathematical model was used. A set of $n$ symbols to be transmitted is represented by the vector $\mathbf{s}=\left[\begin{array}{ll}\mathrm{s}_{0} & \mathrm{~s}_{1} \ldots \mathrm{s}_{\mathrm{n}}\end{array}\right]$. These symbols are then encoded using the Alamouti space-time code to provide a set of two symbol streams, $\mathbf{s}_{\mathbf{a}}$ to be sent out of the transmit antennas.

$$
S_{a}=\left[\begin{array}{cc}
s_{0} & S_{1} \\
-s_{1}^{*} & s_{0}^{*} \\
\vdots & \vdots \\
-s_{n}^{*} & s_{n-1}^{*}
\end{array}\right]
$$

In order to allow for matched filtering at the receiver, the samples are shaped via an interpolating root raised cosine (RRC) filter, $h$, with an excess bandwidth of 0.35 which allows for maximum likelihood detection (MLD) at the receiver and interpolates them to a total of $p$ samples per symbol. This is modelled by convolution of $S_{a}$ with $h$. With the space time coding and the transmit portion the matched filtering complete, the symbols are ready to be transmitted over a simulated channel.

The transmission of the set of symbol $\mathbf{s}_{\mathbf{a}}$ through a fading channel is represented through the multiplication by a vector H containing the two complex coefficients representing the two zero mean Gaussian, independent, identically distributed (ZMGIID) channels between the transmit antennas and the receive antenna. Additionally, the channel includes

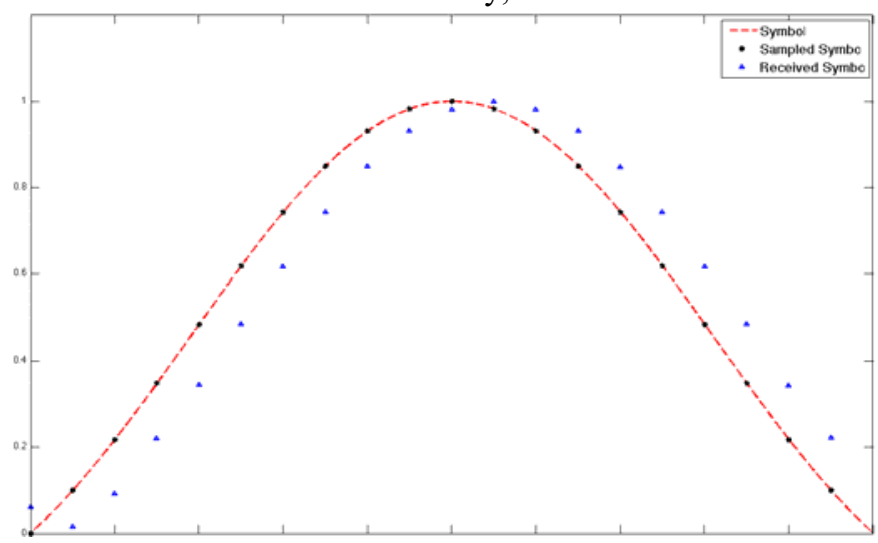

Figure 1: Sampling Offset Example 

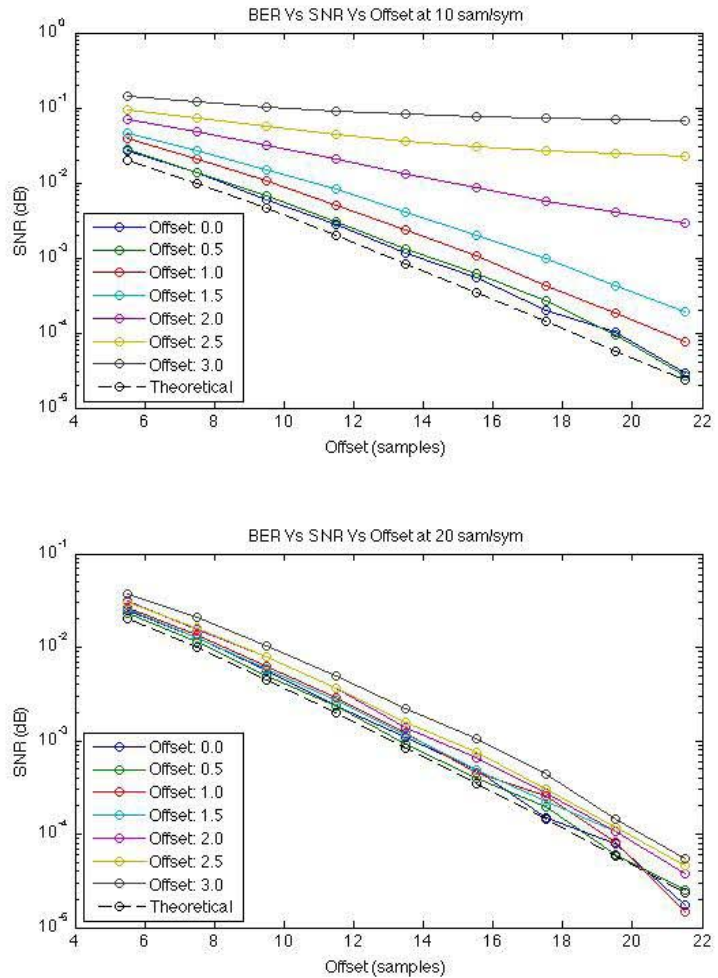
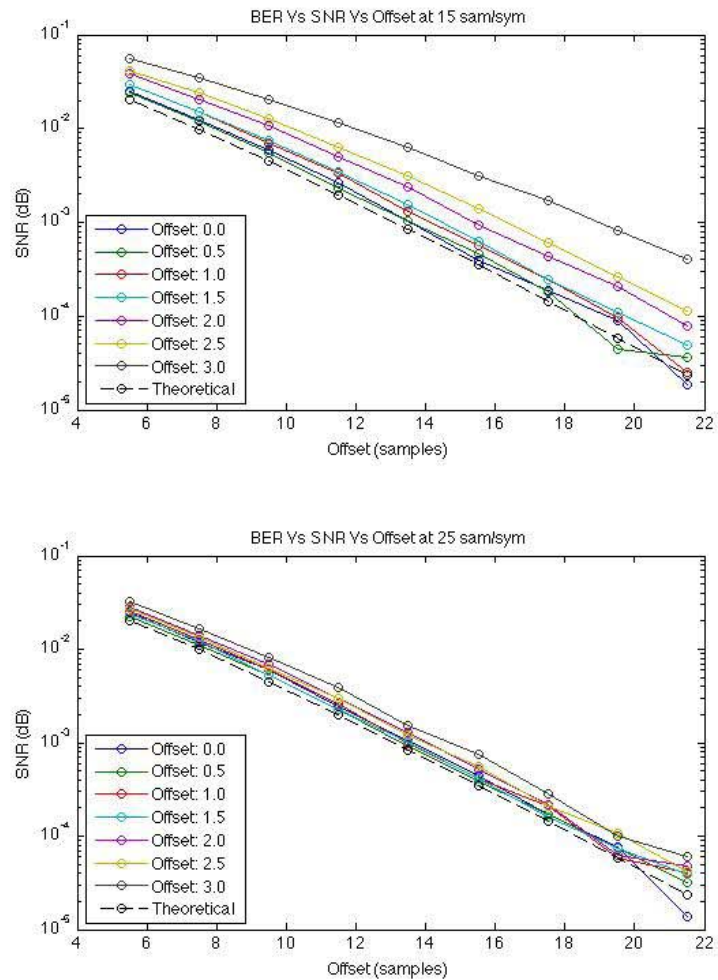

Figure 2: BER Vs SNR for various samples per symbol

additive white Gaussian noise (AWGN), providing for a final representation of the signal at the receiver:

$$
y=\left(s_{a} * h\right) H+n
$$

At the receiver this signal $y$ is sent through a second filter, $h$ ', matched to the transmit filter and is sampled at the center of the symbol. An increasing offset from the center of the symbol is introduced during simulation to represent a discrepancy between the sample clocks of the transmitter and receiver. Because the matched filter receiver relies on sampling at the center of the symbol, an offset between the transmitter and receiver sample clocks introduced by less than ideal timing recovery can lead to an increase in the BER as the correlator output decreases to the noise floor. In order to estimate the impact of the offset, it is necessary to delay the received signal by some amount $\tau$ equal to the delay prior to decoding. The signal that is then sent through the matched filter decoder is specified by

$$
y_{\tau}=\left[\left(s_{a} * h\right) H+n\right] \delta(\tau)
$$

When this signal, $y_{\tau}$, is sent through the decoder at the receiver, additional errors will be introduced depending on the length of the delay. Figure 1 shows the impact of the delay for $\tau$ equal to $1 / 2$ of a sample period. It is clear that the matched filter will not provide an optimum output because of this delay.

\section{SIMULATION MODEL}

By simulating the effects of offsets in sample timing we can analyze the impact that various changes in the development of a communications system can have on BER. By understanding the scope of the changes, it is possible to design a system that utilizes tradeoffs in terms of cost, speed, and reliability in order to provide the best BER at the minimum cost.

For the results presented here, rates of 5, 10, 15, 20, and 25 samples per symbol were tested. The number of samples per symbol used in a communications system can affect the ability of the receiver to accurately reconstruct the transmitted symbols. By increasing the number of samples a receiver has for a given symbol, the approximation of the discrete sampled symbol approaches that of the continuous symbol, allowing more accurate analysis during correlation and maximumlikelihood schema. The trade off in this case is that for a fixed analog to digital converter (ADC) rate, increasing the number of samples for each symbol also increases the amount of time each symbol takes to transmit, thereby reducing the overall data rate. 

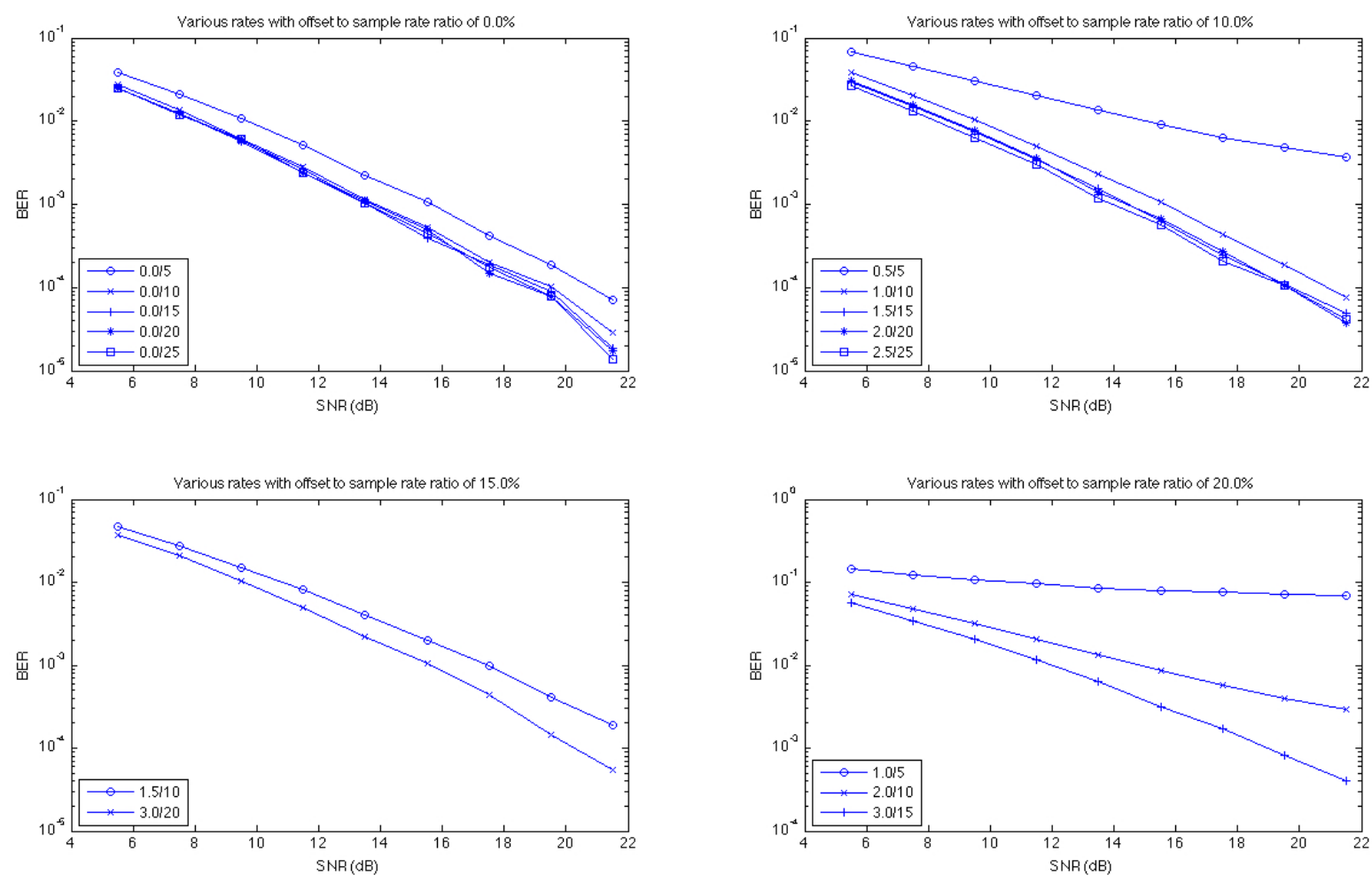

Figure 3: Different Ratios of offset to samples per symbol

In addition to varying the number of samples per symbol, the offset from the center of the symbol is also varied. Because the model represents a discrete time system, the offset is specified as a number of samples, rather than as specific delay $\tau$. Symbol offsets mainly arise from a combination of two effects. First, it is likely that the sample clocks of the transmitter and receiver are not synchronised with respect to one another. This may mean that the received symbol is sampled in between the interpolated discrete points of the symbol generated at the transmitter. Because of this, there may be some fractional sample offset between the received symbol and the ideal symbol sent from the transmitter. The second source of offsets may come from non-ideal symbol timing estimators at the receiver. The most common forms of symbol timing estimation, maximumlikelihood, training symbols, and early/prompt/late schema all suffer from a chance that the estimate of the symbol center will be off by a sample or more depending on the SNR at the receiver. The combination of these two effects are mostly indistinguishable and are represented in the simulation as a single offset from the ideal center of the symbol. Symbol offsets of $0,0.5,1.0,1.5,2.0,2.5$, and 3.0 were considered during simulation. For integer offsets, these timing recovery errors are introduced by shifting the received blocks of symbols by a number of samples equal to the offset. When fractional offsets are considered, the symbols undergo rational resampling in the channel model. That is, they are interpolated by the denominator of the offset, shifted by the numerator, and then decimated back to the original symbol length. It should be pointed out that each symbol offset is positive; the samples in each symbol are advanced by the offset during the channel modelling. It might be more realistic to randomly distribute positive and negative offsets, however, when the RRC pulse shaping is applied, it can be seen that a positive or negative delay has the same effect on a per symbol basis due to the symmetry in the shaped filters. Additionally, each symbol is delayed by the maximum amount in each simulation, providing for a worst case scenario given a maximum allowable delay.

In order to create a simulation environment that would be applicable to modern designs, the model was based on the structure of an $802.11 \mathrm{n}$ system. The packet length, format, and training sequences were used in the simulation. Additionally, the same assumptions regarding block fading were made; specifically that the channel would not change during each block.

\section{SimUlation RESUlTS}

Figure 2 shows the simulation results for the cases where the samples per symbol are 10, 15, 20, and 25. Several conclusions can be drawn from analysis of these graphs. First, it is clear that incorrect sample timing at the receiver leads to a lower bound on the BER of a space-time coded system. Second, there seems to be a non-linear relationship between 
that lower bound and the two parameters, samples per symbol and offset. It can be seen that the samples per symbol and the offset appear to independently affect the BER of the system, in addition to the expected independent variable, the ratio between them. Figure 3 shows the BER curves for various simulations where the ratio of the offset to the number of samples in each symbol is the same. It is easy to see in the graphs that there is a significant difference between the curves despite the ratio being the same.

This gives a space-time system designer some insight into the effects of different parameters when creating a system. For example, it is clearly beneficial, in terms of efficiency, to devote more resources increasing the number of samples per symbol rather than to attempt a reduction in the sample offset error. However, this does not mean that it is always beneficial to maximize the size of each symbol. It can be seen from Figure 3(b) that when the symbol offset error is low (around $10 \%$ ), that there is very little gain to be had by increasing the number of samples per symbol. This implies that the optimum number of samples per symbol needed to account for a given offset also has an upper bound past which increasing it will no longer provide you with noticeable performance gains.

\section{EMPIRICAL MODEL}

Based on the simulation results from Section IV, a system of equations designed to fit the BER curves and allow for accurate estimation of the increase in BER for a given offset at a given number of samples per symbol.

Because only the additional errors introduced by the sampling offset are of interest, the first step is to subtract out the BER of a theoretical Alamouti system. Using this data, the additional BER introduced though unaligned sampling, we can fit to an equation using standard linear regression techniques. Using a simple least squares regression, it was easy to see that the BER added to a theoretical Alamouti system was directly dependent on the SNR. The data could be very accurately fit to the following equation

$$
a \cdot z^{-b}+c
$$

where $z$ is the SNR, $c$ is the BER floor introduced by the offset and $a$ and $b$ affect the rate at which the BER approaches the floor. All 3 coefficients were affected very strongly by the ratio between the offset and the samples per symbol, but as discussed in section IV, this alone was not enough to

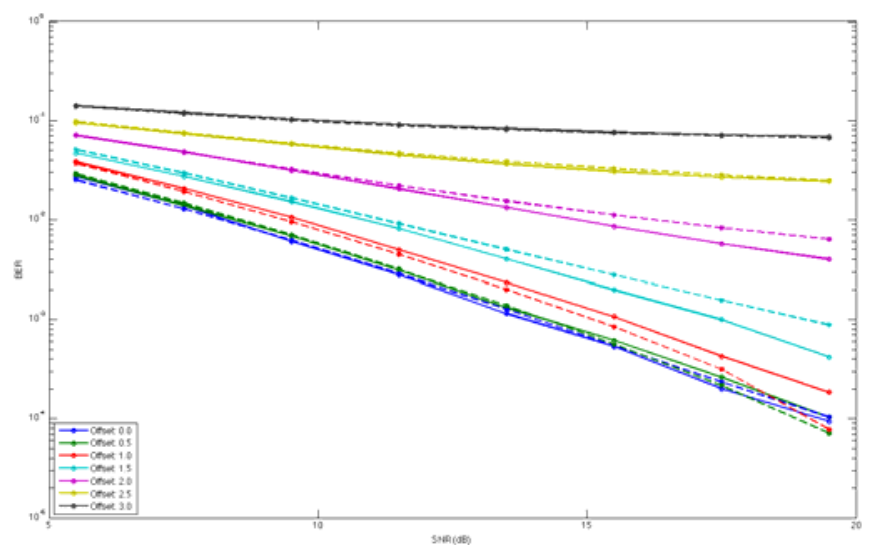

accurately represent the system. In addition, offset and samples per symbol terms were required to approximate the additional BER.

Both of the $a$ and $b$ coefficients were fit to a simple summation of the first, second, and third powers of each of the three variables as follows

$$
\begin{aligned}
F= & a_{1} \cdot s+a_{2} \cdot s^{2}+a_{3} \cdot s^{3}+ \\
& a_{4} \cdot o+a_{5} \cdot o^{2}+a_{6} \cdot o^{3}+ \\
& a_{7} \cdot \frac{o}{s}+a_{8} \cdot \frac{o^{2}}{s}+a_{9} \cdot \frac{o^{3}}{s}+a_{10}
\end{aligned}
$$

where $o$ is the offset, $s$ is the number of samples per symbol, and $a_{\mathrm{n}}$ is the $n^{\text {th }}$ coefficient of the fit. This fit allows for a flexible interaction between the main component, the ratio of the offset to the number of samples per symbol, and the variables it is dependent on.

The final parameter to be fit, $c$, is the floor that the BER approaches as the SNR increases. This floor exists in all nontheoretical systems, and stems from the fact that with imperfect channel estimation, timing recovery, etc, it will be impossible to reach certain theoretical BERs. Though these floors exist, their bounds are not often tested due to the fact that channel simulations will typically rely on perfect channel knowledge and timing synchronization. In this case, the error floors are clearly apparent for some cases, and easy to extrapolate for others. This floor can be shown to be dependent on the offset $(o)$ and number of samples per symbol $(s)$ as follows

$$
F=a_{1} \cdot o^{a_{2}}+a_{3} \cdot s^{a_{4}}+a_{5} \frac{o}{s}^{a_{6}}
$$

With the model equations set, each of the coefficients $a_{\mathrm{n}}$ for $a, b$, and $c$ can be found traditional linear regression techniques. These calculated coefficients are in Table 1 at the end of the paper.

Figure 4 shows the simulated BERs along with the values generated by the empirical model. It is clear that this model is particularly effective when the offset is small, $5-10 \%$ of the number of samples. Additionally the model is very accurate when the offset is very high with respect to the number of

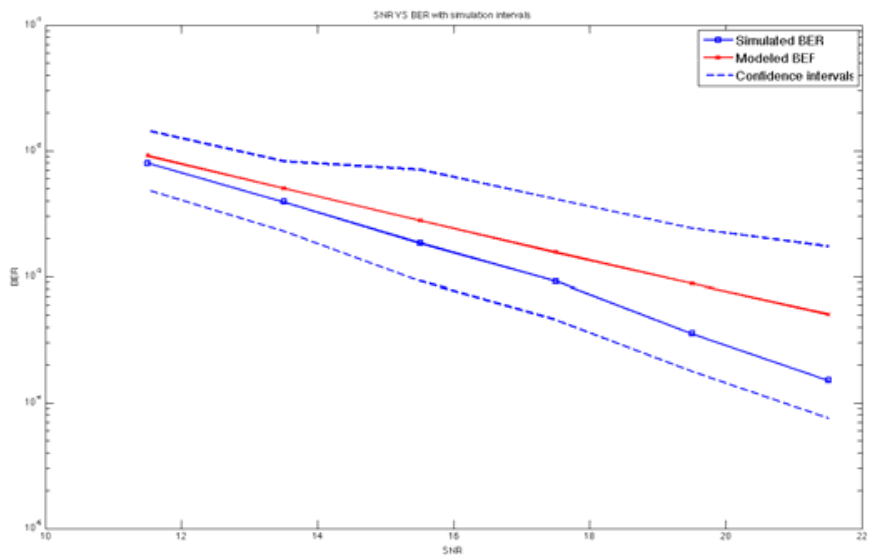

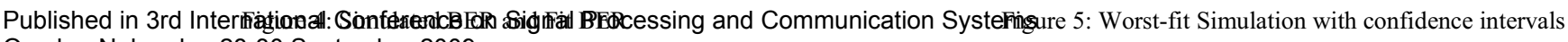
Omaha, Nebraska, 28-30 September 2009. 
samples $(25 \%+)$. This is because the BER floor $(c)$ has a greater impact on the fit with respect to the impact of the SNR.

Even with the divergence of the fit from the simulated data, it can be seen from Figure 5 that the data falls within the expected confidence intervals of the simulation. Figure 5 shows the BER curve with the worst mean residuals after fitting, an offset of 1.5 samples with 10 samples per symbol, along with the $95 \%$ confidence interval of the simulation. It can be seen that even at its worst, the empirical model falls within these $95 \%$ bounds.

\section{CONCLUSIONS}

In this paper we presented an initial investigation into the effects of errors in sample timing on the bit error rate of a space-time coded system in a flat fading environment. Initial results confirm the logical conclusion that as the estimated symbol center drifts from the actual center of the symbol that the BER of the system increases. The more interesting results of the investigation show that there is an independent, nonlinear relationship between the offset and the number of samples per symbol with respect to the BER of the system. This relationship is modelled by fitting the empirical data to an equation for easy estimation, and the results give insight into the tradeoffs that a designer may make when developing a space-time coded system. When designing a space-time coded system, therefore, it is more beneficial to devote resources to increasing the number of samples per symbol rather than refining the timing estimate. Additionally, there are clearly bounds on both the BER for a given number of samples per symbol, as well as the number of samples required to negate a given offset size.

TABLE 1: FIT COEFFICIENTS

\begin{tabular}{|c|c|c|c|}
\hline & $a$ & $b$ & $c$ \\
\hline $\mathrm{a}_{1}$ & $-3.3388 \mathrm{e}-05$ & $5.6657 \mathrm{e}-05$ & $-1.3622 \mathrm{e}-04$ \\
\hline $\mathrm{a}_{2}$ & $1.5695 \mathrm{e}-03$ & $-3.7582 \mathrm{e}-04$ & $1.6830 \mathrm{e}+00$ \\
\hline $\mathrm{a}_{3}$ & $-9.1463 \mathrm{e}-03$ & $-1.0193 \mathrm{e}-01$ & $2.5810 \mathrm{e}-09$ \\
\hline $\mathrm{a}_{4}$ & $6.6216 \mathrm{e}-03$ & $-6.6188 \mathrm{e}-02$ & $3.6926 \mathrm{e}+00$ \\
\hline $\mathrm{a}_{5}$ & $1.8473 \mathrm{e}-04$ & $3.4661 \mathrm{e}-01$ & $6.4456 \mathrm{e}+02$ \\
\hline $\mathrm{a}_{6}$ & $-2.0971 \mathrm{e}-01$ & $-2.5740 \mathrm{e}-01$ & $7.7760 \mathrm{e}+00$ \\
\hline $\mathrm{a}_{7}$ & $-5.8369 \mathrm{e}+00$ & $-2.5051 \mathrm{e}+01$ & - \\
\hline $\mathrm{a}_{8}$ & $-4.3513 \mathrm{e}-01$ & $-1.0015 \mathrm{e}+01$ & - \\
\hline $\mathrm{a}_{9}$ & $2.1307 \mathrm{e}+00$ & $9.9820 \mathrm{e}+00$ & - \\
\hline $\mathrm{a}_{10}$ & $1.0110 \mathrm{e}-01$ & $-1.4948 \mathrm{e}+00$ & - \\
\hline
\end{tabular}

\section{REFERENCES}

[1] Paulraj etal (2003). Introduction to Space Time Wireless Communications. Cambridge University Press, United Kingdom

[2] Alamouti, S. (1998). A simple transmit diversity technique for wireless communications. Selected Areas in Communications, IEEE Journal on $16(8), 1451-1458$.

[3] Danesfahani, G., \& Jeans, T. (1995). Optimisation of modified Mueller and Muller algorithm. Electronics Letters , 31 (13), 1032 - 1033.

[4] Dmochowski, P., \& McLane, P. (2009, Jul 6). On the Properties of a Robust Timing Error Detector for Alamouti Space-Time Coding in Rayleigh Fading MIMO Channels with Randomly Distributed Timing Drift. 1-6.

[5] Dmochowski, P., \& McLane, P. (2005). Robust timing epoch tracking for Alamouti space-time coding in flat Rayleigh fading MIMO channels. 2005 IEEE International Conference on Communications, 2005. ICC $2005,4$.

[6] Harris, F., \& Rice, M. (2001). Multirate digital filters for symbol timing synchronization in software defined radios. Selected Areas in Communications, IEEE Journal on , 19 (12), 2346 - 2357.

[7] Kyung Seung Ahn, \& Heung Ki Baik. (2004). Training-based channel estimation and equalization for space-time block-coded systems over frequency-selective fading channels. Vehicular Technology Conference, 2004. VTC2004-Fall. 2004 IEEE 60th , 3, 1748 - 1752 Vol. 3.

[8] Nelson, T., \& Rice, M. (2003). Alamouti Space-time Coding for QPSK with Delay Differential. Proceedings of the International Telemetry Conference, 1249-1258.

[9] Wu, Y., \& Chan, S. (2003). On the symbol timing recovery in spacetime coding systems. 2003 IEEE Wireless Communications and Networking, 2003. WCNC 2003, 1 . 